DOI: https://doi.org/10.46296/yc.v5i9edespsep.0100

\title{
EL ABP COMO METODOLOGÍA CENTRAL EN EL PROGRAMA DE PARTICIPACIÓN ESTUDIANTIL
}

\section{PBL AS A CENTRAL METHODOLOGY IN THE STUDENT PARTICIPATION PROGRAM}

\author{
Delgado-Moreira Lenin Antonio ${ }^{\text {; }}$ Game-Varas Cinthya Isabel ${ }^{2}$ \\ ${ }^{1}$ Universidad San Gregorio de Portoviejo USGP. Portoviejo Ecuador. \\ lenindelgadomoreira@gmail.com ORCID: https://orcid.org/0000-0003-0194-4516 \\ 2 Universidad San Gregorio de Portoviejo USGP. Portoviejo Ecuador. \\ cinthya.gamev@gmail.com.ec ORCID: https://orcid.org/0000-0002-4877-1192
}

\begin{abstract}
Resumen
Esta investigación identifica la aplicación de la metodología del aprendizaje basado en proyectos (ABP) dentro del programa de participación estudiantil (PPE), implementado a nivel nacional a través del Ministerio de Educación para los estudiantes de primero y segundo de bachillerato, el estudio tuvo un enfoque cualitativo, de tipo exploratorio - descriptivo, se realizó una revisión bibliográfica sobre esta metodología, se utilizó dos métodos: el analítico y el deductivo, y se utilizó la técnica de una entrevista semiestructurada aplicada a docentes facilitadores responsables del programa. De los resultados obtenidos, se destaca que, para la mitad de docentes entrevistados, los proyectos, contribuyen en la formación del pensamiento crítico, la creatividad y personalidad de los estudiantes para abordar situaciones, que pueda presentarse dentro de su entorno socioeducativo, sin embargo, presentaron dificultades para adaptar el ABP a los ambientes virtuales, y elaboran planificaciones desatendiendo el aprendizaje colaborativo, estando direccionadas al cumplimiento de actividades aisladas. Se concluye, que los docentes aplican el ABP con falencias en el dominio de esta metodología; además, los docentes aplican fielmente las instrucciones de la guía metodológica del Ministerio de Educación del Ecuador.
\end{abstract}

Palabras claves: ABP, aprendizaje, metodología, participación estudiantil, programa educativo.

\begin{abstract}
This research identifies the application of the project-based learning (PBL) methodology within the student participation program (PPE), implemented nationally through the Ministry of Education for first and second year high school students. The study had a qualitative, exploratory-descriptive approach, a literature review was conducted on this methodology, and two methods were used: analytical and deductive, and the technique of a semi-structured interview applied to teacher facilitators responsible for the program was used. From the results obtained, it is highlighted that, for half of the teachers interviewed, the projects contribute to the formation of critical thinking, creativity and personality of the students to address situations that may arise within their socioeducational environment, however, they presented difficulties in adapting PBL to virtual environments, and elaborate planning neglecting collaborative learning, being directed to the fulfillment of isolated activities. It is concluded that teachers apply PBL with deficiencies in the mastery of this methodology; in addition, teachers faithfully apply the instructions of the methodological guide of the Ministry of Education of Ecuador.
\end{abstract}

Keywords: PBL, learning, methodology, student participation, educational program.

Información del manuscrito:

Fecha de recepción: 29 de junio de 2021.

Fecha de aceptación: 01 de septiembre de 2021.

Fecha de publicación: 09 de septiembre de 2021. 


\section{Introducción}

Esta investigación, tiene su origen en la observación y exploración de la metodología de aprendizaje basado en proyectos ( $A B P)$, que se aplica dentro del programa de participación estudiantil (PPE), durante el año lectivo 2020 - 2021, que, debido a la pandemia mundial, las aulas de clases fueron trasladadas a los hogares de cada estudiante.

Aunque esta metodología del ABP, "se desarrolló hacia finales del siglo XIX, y tomó más relevancia en las aulas del Siglo XX y XXI" (UNICEF, 2020, pág. 9). Internacionalmente hay dificultades en su aplicación, como lo indica García \& Basilotta (2017), en que "el ABP es un reto para docentes, pues necesitan apoyo para planificar y difundir el $A B P$ con eficacia, mientras que los estudiantes necesitan ayuda para organizar su tiempo, completar tareas, e integrar la tecnología en los proyectos de manera significativa" (pág. 115). Dificultades que también se presentan en las aulas ecuatorianas con respecto a la aplicación de esta metodología.

Consecuentemente, en el Ecuador también se exteriorizan otras dificultades, como concluyó Tandazo (2014) de la Universidad San francisco de Quito, que "en cuanto al $A B P$, los alumnos están enfrentados a situaciones en que deben resolver problemas, crear productos, 0 producir explicaciones, y esto no se logra a través de una sola asignatura, pues requiere de dos 0 más asignaturas" (pág. 50). Por lo que es necesario la colaboración de otros docentes de asignaturas en los proyectos del programa de participación estudiantil, o que los docentes facilitadores del programa dominen diversos campos de conocimientos para la aplicación de esta metodología.

Además, el objetivo del presente estudio fue identificar como los docentes facilitadores del programa del PPE, aplicaron bajo estas circunstancias la metodología del ABP a los estudiantes de 1 ro y 2 do de Bachillerato, Programa que permite respuestas efectivas a las problemáticas sociales reconocidas por los estudiantes, y consecuentemente, que los resultados de esta investigación ayude a las demás instituciones del Ecuador, ya que, el PPE se implementa en todo el territorio 
nacional (MINEDUC-Acuerdo

Ministerial, 2016).

\section{Aplicación de la metodología del} aprendizaje basado en proyectos (ABP).

A nivel mundial, el ABP es adaptado de forma muy diversa "ha pasado de ser un método de trabajo didáctico en aulas con número reducido de estudiantes (...) hasta una propuesta de aprendizaje autónomo con 60130 estudiantes" (Delgado, 2015), Además, se trajo a consideración sobre este estudio: Ia Tesis Doctoral de Verónica Basilotta Gómez-Pablos (2018), donde se concluye que "los docentes consideran que en muchas ocasiones no disponen de la infraestructura tecnológica necesaria, sin embargo, esto no es un impedimento para desarrollar prácticas innovadoras en los centros" (Basilotta, 2018, pág. 591).

Por otra parte, Bautista, et al, (2017), indican que "es de notable importancia que los profesores y los alumnos implicados en este modelo, estén totalmente familiarizados con el contenido y con el proceso metodológico que se pretende seguir para ir alcanzando las diferentes etapas o fases establecidas" (pág.
53). Por lo tanto, se define el ABP, como "un paradigma o estrategia de aprendizaje, una metodología de enseñanza y una metodología o modelo de trabajo" (Herrera, et al, 2019). Y que, "su principal característica es que promueve el trabajo en equipo entre docentes y estudiantes sobre temas reales previamente seleccionados" (pág. 15)".

En ocasiones se puede confundir el ABP de proyectos con el ABP de problemas y "aunque ambas metodologías están estrechamente relacionadas, no son idénticas" (Basilotta, 2018). "El ABP de proyectos pone énfasis en el producto final y en las habilidades adquiridas durante el proceso, mientras que el ABP de problemas tiene como objetivo prioritario la búsqueda de soluciones a los problemas identificados" (pág. 166).

Condiciones fundamentales para aplicar la metodología ABP.

En la actualidad, el proceso de enseñanza- aprendizaje a distancia, ha empezado a incursionar en diferentes ámbitos pedagógicos, por tal motivo, hay condiciones fundamentales para aplicar la 
metodología del ABP y el trabajo en equipo es esencial para la metacognición de los estudiantes, ya que, dentro de esta metodología, cada estudiante aporta y asimila conocimientos al equipo durante la reflexión y la autonomía que implica el ABP.

Además, "en las clases en donde se trabaja por proyectos, el ambiente tiene que tener unas características particulares que se parecen más a lo que conocemos como taller que a la idea que tenemos de clase tradicional" (UNICEF, 2020, pág. 43). $Y$ el objetivo del ABP se lo puede plantear como indica Lucero (2016), que "es contrarrestar aprendizajes memorísticos y enseñar al estudiante trabajo cooperativo con la ayuda de la interdisciplinariedad" (pág. 1). Adicional Basilotta (2018), resume el $A B P$ en tres fases siguientes:

Fase de planificación, donde se elige el tema, se organizan los materiales necesarios, las tareas, los tiempos y los pasos necesarios para la ejecución del proyecto. Fase de creación e implementación, donde el alumno desarrolla la idea del proyecto, combina sus ideas con las aportaciones del grupo, y trabaja en el producto final. La fase de procesamiento incluye la reflexión y el seguimiento del proyecto. En esta etapa los alumnos comparten sus productos (trabajos realizados) en grupos o con toda la clase, obtienen retroalimentación $y$ reflexionan sobre el proceso de aprendizaje realizado. (págs. 168-169).

No obstante, para Bautista, et al, (2017), "la metodología Aprendizaje Basado en Proyectos parece prometedora, por cuanto permite convertir en proyectos todos los contenidos científicos $\mathrm{y}$, consecuentemente, permite que los alumnos sean capaces de aplicar los conocimientos en el mundo real" (pág. 44). Consecuentemente, es posible que el ABP se aplique en todas las áreas del conocimiento dentro de los procesos de enseñanza aprendizaje. Sin embargo, "los escenarios educativos actuales requieren de una nueva mirada que permita la introducción del Aprendizaje Basado en Proyecto de manera interdisciplinar, de modo tal, que estimule al estudiante a convertirse en protagonista de su 
aprendizaje" (Medina \& Tapia , 2017 , pág. 245).

\section{Programa de Participación Estudiantil (PPE).}

El Ministerio de Educación implementa el PPE en todas las instituciones educativas del territorio ecuatoriano, independientemente del sostenimiento al que pertenezca, fiscal, fisco-misional o particular; y que oferten el bachillerato. Este programa es obligatorio para los estudiantes de primero y segundo de bachillerato cumpliendo 200 horas dentro del mismo, como requisito para la obtención del título de bachiller y la metodología base de este programa es el aprendizaje basado en proyectos (MINEDUCAcuerdo Ministerial, 2016).

Así mismo, como indica la guía metodológica para docentes facilitadores del PPE, el programa se relaciona con el perfil de salida del bachillerato ecuatoriano (justos, innovadores y solidarios) "puesto que es una oportunidad de aprendizaje para los y las estudiantes que fortalece el trabajo en equipo" (MINEDUC, 2019),
$Y$ estableciendo que, un estudiante realiza dos proyectos para su comunidad antes de su graduación, en grupos de 50 alumnos por cada docente facilitador del programa, para Hidalgo \& Perines (2018), "la gran mayoría del profesorado es enfática al defender que una de sus prioridades es fomentar la participación estudiantil" (pág. 10). Y realmente, la consolidación de la participación estudiantil en el desarrollo del PPE dentro de las unidades educativas, es uno de los retos que tienen los maestros actualmente.

Las fases del PPE según el instructivo para la implementación del programa de participación estudiantil son 4, y deben completarse en cada año del bachillerato en que se implementa el programa. Estas fases son las siguientes: "Inducción, donde socializan parámetros, metodología y fases; Planificación, se elabora el proyecto educativo interdisciplinario por parte de los estudiantes; Implementación, se ejecutan las actividades planificadas dentro del proyecto, conforme al cronograma; $y$ Evaluación, que evalúa al estudiante y al proyecto" (MINEDUC, 2019). 


\section{Método}

La investigación tuvo un enfoque cualitativo, el mismo que consideró la identificación de los factores que se presenta en la implementación del aprendizaje basado en proyectos ABP, durante el desarrollo del programa de participación estudiantil - PPE. Además, se realizó una revisión bibliográfica sobre esta metodología, donde se describieron características de la misma sobre el programa del PPE. Por consiguiente, la investigación se desarrolló bajo un enfoque cualitativo de tipo exploratorio - descriptivo.

En la investigación se utilizó dos métodos el analítico y el deductivo. El método analítico fue pertinente en la investigación, porque describió los resultados alcanzados desde las particularidades de las condiciones en que se aplica el ABP dentro del PPE. Mientras que, el método deductivo estableció los efectos que tienen los lineamientos generales presentes en el PPE, en situaciones específicas en la aplicación interdisciplinaria de la metodología del ABP dentro del programa desarrollado en la unidad educativa "4 de Noviembre" de la ciudad de Manta, Ecuador.
Consecuentemente, para la investigación, la técnica utilizada fue una entrevista semiestructurada aplicada en forma individual a los 12 docentes facilitadores del programa de participación estudiantil. La población objeto de este estudio, constituyó el grupo de docentes de la unidad educativa fiscal "4 de Noviembre" que aplican la metodología del ABP dentro del programa de participación estudiantil en el primer y segundo año del bachillerato general unificado, durante el segundo semestre del año 2020.

\section{Resultados}

Para 5 docentes del total de la población de entrevistados, el aprendizaje basado en proyectos es solo una metodología, "porque se basa directamente en la parte del estudiante, se centra en que el principal actor de todo es el estudiante" E2. Sin embargo, el ABP se define como "un paradigma o estrategia de aprendizaje, una metodología de enseñanza y una metodología o modelo de trabajo" (Herrera, et al, 2019, pág. 15). 
Otra categoría de esta metodología, es que el estudiante es el centro del proceso de enseñanza-aprendizaje, 10 docentes de la población en estudio expresaron que al planificar sus actividades hacen énfasis en la motivación y participación activa del estudiante. "Es lo que yo más busco, que participen activamente del tema que se está hablando, que sea de provecho para que lo puedan llevar a la vida práctica" E1. Sin embargo, Basilotta (2018) indica que, "EI ABP de proyectos pone énfasis en el producto final y en las habilidades adquiridas durante el proceso" (pág. 166).

El ABP actualmente debe ser adaptado dentro de los ambientes virtuales, y 6 de 12 docentes entrevistados, indicaron que, en su lugar aplicaron la guía metodológica del docente facilitador del programa de participación estudiantil proporcionada por el Ministerio de Educación del Ecuador, "realmente toda esta situación virtual ha sido bien complicada, se nos ha complicado a todos los maestros, en este asunto de la parte virtual, se tenía una guía que estaba dividida en varios momentos" E11. No obstante, "en las clases en donde se trabaja por proyectos, el ambiente tiene que tener unas características particulares que se parecen más a lo que conocemos como taller que a la idea que tenemos de clase tradicional” (UNICEF, 2020, pág. 43).

Respecto al proceso de evaluación dentro del programa del PPE, 7 docentes del total de entrevistados indican que está basado solo en el cumplimiento de las actividades que los estudiantes realizan de manera individual desde casa. "En este caso tampoco había un proceso de evaluación reconocido porque se evaluaba o calificaba simplemente el cumplimiento de las actividades que se hacía durante la semana" E3. A pesar que, "se considera como un factor imprescindible de la autorregulación metacognitiva exitosa, la presencia de la actividad cooperativa y la colaboración del grupo en la construcción de significados y resolución de problemas que no lograría el estudiante promedio trabajando aisladamente" (Flórez, 2000, pág. 9).

La población docente entrevistada para este estudio, no se identifican con ningún autor de la metodología del aprendizaje basado en proyectos, para el seguimiento 0 
aplicación de las fases que pueden definirse dentro del ABP. "En mi caso seguía la guía del estudiante que nos envía el Ministerio de Educación siguiendo las actividades que están ahí" E3. Giménez (2016), resume el ABP en tres fases siguientes: "Presentación del proyecto, donde se define el problema. Planteamientos de retos asumibles, donde los alumnos plantean investigaciones para concretar y resolver problemas. Desarrollo y resolución de proyecto, donde se soluciona el problema y se presentan los resultados" (pág. 23).

Asimismo, para la planificación de las actividades, 7 docentes entrevistados las elaboran en solitario, sin incluir a otros docentes inmersos en el proyecto. "En sí era una cuestión más individual porque nunca nos reuníamos para planificar, solamente para ver indicaciones del maestro coordinador de PPE". E5 Y 10 de ellos, indicaron la facilidad del área de ciencias naturales para aplicar la metodología del ABP, "En mi opinión es más fácil aplicar el ABP en ciencias naturales" E1, así como el área de educación cultural artística y de ciencias sociales que también tienen un alto grado de facilidad.
La fortaleza del ABP se ve evidenciado cuando, 6 de la totalidad de docentes entrevistados opinan que el conocimiento se amplía por la interdisciplinariedad de las diferentes asignaturas. "El estudiante se ve inmerso en saber de todo un poco, es decir, inmiscuirse en el saber de todas las áreas, no solamente se enfoca en una asignatura, inclusive esto le ayuda a tener más competencia en muchos aspectos" E11. Mientras que la otra mitad, considera que los proyectos ayudan o contribuyen en la formación del pensamiento crítico, la creatividad y personalidad de los estudiantes, para asumir o abordar futuras situaciones que pueda presentarse dentro de su entorno socioeducativo. "Esta manera de proyecto a desarrollar por el estudiante, es que ellos pueden con un solo tema o una sola actividad desarrollar mucho su pensamiento crítico" E10. Por lo tanto, el ABP de proyectos si se está implementando, porque las características de este aprendizaje son evidenciadas por los maestros.

La aplicación de la herramienta cualitativa nos permite tener directamente de la población 
entrevistada sugerencias de

aplicabilidad del ABP dentro del PPE, entre ellas están que el programa sea práctico, que tenga una calificación cuantitativa, además que, no se utilicen las horas del programa de participación estudiantil como relleno de carga horaria para los docentes de la institución, y que el estudiante pueda elegir su propio campo de acción en la aplicación de los proyectos del PPE. Estas premisas que los docentes formulan, se instituyen "como fundamento para el desarrollo de competencias y la mejora de la calidad del proceso de enseñanza-aprendizaje" (Medina \& Tapia , 2017, pág. 245). Por lo tanto, lo que describen los docentes, son consideraciones oportunas para el entorno del estudiante y que pretenden en buena parte, mejorar este programa basado en el ABP.

\section{Conclusiones}

Se identifica que los docentes investigados, aplican el aprendizaje basado en proyectos sin tener un dominio de la metodología, por lo tanto, no existe una visualización homogénea del ABP como estrategia dinámica metodológica y guía de trabajo a seguir por parte de los docentes entrevistados del programa de participación estudiantil. El énfasis de sus planificaciones no se centra en las habilidades de los estudiantes, sino en la participación y cumplimiento del estudiante.

Se concluye que, los docentes se limitan dentro del programa de participación estudiantil a seguir únicamente con las indicaciones de la guía metodológica del docente facilitador del programa que otorga el Ministerio de Educación del Ecuador, por lo que, el trabajo colaborativo y cooperativo que implica la metodología del ABP queda sesgado debido a esta delimitación.

Se determina que la evaluación dentro del programa, queda confinada a la presentación de actividades, y no, a la discusión y presentación del producto final como parte de la metodología del aprendizaje basado en proyectos en la que se basa el programa de participación estudiantil. Las actividades expuestas en la guía son netamente individuales, las mismas que se convierten en el principal y hasta el único insumo del docente para la evaluación. 
Otro hallazgo es, que los docentes investigados desconocen 0 no indagan sobre la metodología del $A B P, y$ mucho menos de sus fases para realizar el seguimiento del aprendizaje de los estudiantes cuando estos se encuentran en cada una de las etapas, así como de las habilidades adquiridas en cada fase. También, se determina que es muy escaso el enfoque interdisciplinario que los docentes le dan a las actividades planificadas dentro del PPE, ya que su elaboración se realiza de manera individual sin integrar a otros docentes 0 profesionales de la comunidad educativa.

Para los docentes investigados, el desarrollo de proyectos, contribuye en la ampliación del conocimiento, a través del aprendizaje entre pares y el trabajo cooperativo. Estos proyectos, son el complemento que los docentes facilitadores del programa, necesitan motivar y expandir dentro del proceso de enseñanza-aprendizaje para la formación integral de los estudiantes.

La investigación aporta, la necesidad de formación y/o actualización del docente, lo cual es importante para que el $A B P$ se extienda por todas las áreas del conocimiento, ya que, los docentes investigados en su mayoría, expresan la facilidad de aplicar proyectos desde cualquier área del conocimiento, principalmente el área de las ciencias naturales como un campo generalizado para aplicarlos dentro del programa de participación estudiantil.

\section{Bibliografía}

Basilotta, V. (2018). El valor del aprendizaje basado en proyectos con tecnologías: Análisis de prácticas de referencia. Salamanca, España: Universidad de Salamanca.

Bautista, J., Espigares, M., \& Hernandez, R. (2017). Aprendizaje Basado en Proyectos $(\mathrm{ABP})$ ante el reto de una nueva enseñanza de las ciencias. Revista Brasileira de Ensino de Ciência e Tecnologia, 10(3), 43-60, ISSN:1982-873X. doi:10.3895/rbect.v10n3.4454

Delgado, K. (2015). Aprendizaje colaborativo. Teoría y práctica.

ISBN: 9789582012076. Málaga, España: Magisterio, Cooperativa Editorial. 
Flórez, R. (2000). Autorregulación, metacognición y evaluación. Acción Pedagógica, 9(1 Y 2), 4-11, ISSN-e: 1315-401X. Obtenido de https://dialnet.unirioja.es/servl et/articulo?codigo $=2973266$

García, A., \& Basilotta, V. (2017). Aprendizaje Basado en Proyectos (ABP): evaluación desde la perspectiva de alumnos de Educación Primaria. Revista de Educación Investigativa (RIE), 35(1), 113-131, ISSN: 0212-4068. Murcia, España: Asociación Interuniversitaria de Investigación Pedagógica.

Giménez, E. (2016). Metodología basada en proyectos, aplicación en la asignatura de tecnología de $1^{\circ}$ de bachillerato. Logroño, España: Universidad Internacional de la Rioja.

Herrera, E., Vásquez, W., \& Yuquilima, L. (2019). Estrategia metodológica para fomentar aulas inclusivas a través del Aprendizaje Basado en Proyectos: Un estudio comparativo multicaso. Azogues, Ecuador: UNAE - Universidad Nacional de Educación.

Hidalgo, N., \& Perines, H. (2018). Dar voz a los protagonistas: La participación estudiantil en el proceso de enseñanzaaprendizaje.

Revista Educación, 42(2), 1-19, ISSN:
0379-7082, Costa Rica: Universidad de Costa Rica. doi:https://doi.org/10.15517/r evedu.v42i2.27567

Lucero, N. (2016). Aprendizaje basado en proyectos y el rendimiento académico de los estudiantes de la Unidad Educativa Pasa. Ambato, Ecuador: Universidad Técnica de Ambato.

Medina, M., \& Tapia , M. (2017). El Aprendizaje Basado en Proyectos una oportunidad para trabajar interdisciplinariamente.

Olimpia, 14(46), 236-246, ISSN: 1817-9088. Bayamo, Cuba: Revista de la Facultad de Cultura Física de la Universidad de Granma.

MINEDUC. (2019). Guía metodológica para docentes facilitadores del PPE. Régimen Costa. Obtenido de Ministerio de Educación del Ecuador: https://educacion.gob.ec/recu rsos-del-ppe/

MINEDUC. (2019). Instructivo para la implementación del PPE. Régimen Costa. Obtenido de Ministerio de Educacion del Ecuador:

https://educacion.gob.ec/recu rsos-del-ppe/

MINEDUC-Acuerdo Ministerial. (2016). ACUERDO Nro. MINEDUC-ME-2016-00040-

A. Obtenido de Ministerio de 
Educación del Ecuador: https://educacion.gob.ec/doc umentos-legales-ynormativos/

Tandazo, M. (05 de 2014). La enseñanza de Ciencias Naturales a través del Arte por medio del Aprendizaje Basado en Proyectos. Quito, Ecuador: Universidad San Francisco de Quito.

UNICEF. (2020). El Aprendizaje Basado en Proyectos en PLaNEA Características, diseño, materiales e implementación. Buenos Aires, Argentina: (c) Fondo de las Naciones Unidas. Obtenido de https://www.unicef.org/argenti na/publicaciones-y-datos 